\title{
Ueber die fermentative Eiweissspaltung und Ammoniakbildung in der Leber. ${ }^{1}$ ) \\ Von
}

Dr. Martin Jacoby aus Berlin.

(Aus dem physiologisch-chemischen Institut zu Strassburg. Neue Folge Nr. 34.) (Der Redaction zugegangen am 20. Juni 1900.)

Bei Gelegenheit der Nachprüfung einer Angabe von Richet hat Loewi ${ }^{2}$ ) beobachtet, dass es durch Einwirkung von Leberfermentlösung auf Glycocoll gelingt, eine äther-alkohollösliche Substanz zu gewinnen, welche zwar nicht Harnstoff ist, aber leicht abspaltbaren Stickstoff enthält.

Mit den fermentativen Vorgängen in der Leber beschäftigt, hatte ich die Absicht, die Loewi'sche Beobachtung weiter $\mathrm{zu}$ verfolgen und vielleicht jenen äther-alkohollöslichen Körper näher zu charakterisiren. Die Untersuchung nahm jedoch durch einen zufälligen Befund eine andere Richtung an und führte zunächst zur Auffindung eines Ammoniak bildenden Fermentes in der Leber.

\section{Ueber fermentative Bildung von durch Magnesia austreibbarem Stickstoff in der Leber.}

Da Loewi gefunden hatte, dass seine aus Glycocoll erhaltene äther-alkohollösliche Substanz Stickstoff in ziemlich leicht abspaltbarer Form enthält, so wurde in den ersten Versuchen untersucht, ob nach Digestion von Lebersaft mit Glycocoll mehr beim Kochen mit Magnesia abspaltbarer Stickstoff nachweisbar ist, als wenn man Lebersaft ohne Glycocoll digerirt.

1) Ausgeführt mit Unterstützung der Stiftung der Gräfin Bose.

2) Zeitschr. f. physiol. Chemie, Bd. XXV. 1898. 
In einer Anzahl von Versuchen, auf deren Wiedergabe ich bei ihrem durchaus negativen Ausfall verzichte, gelang es jedoch weder durch Zusatz von Glycocoll zum Lebersaft, noch durch Zusatz von Globulinlösungen, die aus Leber bereitet wurden, noch durch Hinzufügung von Extracten, die durch Kochen von Leber gewonnen wurden, eine Vermehrung des durch Magnesia austreibbaren Stickstoffs zu erzielen. Es fiel jedoch auf, dass mit Toluol einige Zeit conservirter Lebersaft viel höhere Magnesia-Stickstoffwerthe gab, als frischer, obwohl Fäulniss sicher ausgeschlossen war. Versuche mit Chloroformwasser oder solche, bei denen Chloroform und Toluol combinirt wurden, führten zu denselben Resultaten.

Diese Beobachtung wurde nun durch besondere Versuche sichergestellt. Aus zahlreichen mir vorliegenden Protokollen dieser Art gebe ich hier einige wieder:

Versuchsprotokoll. I. Am 31. Januar 1899 Nachmittags 6 Uhr werden 4 gut gefütterte Hunde getödtet. Die sofort herausgenommenen Lebern wiegen zusammen $1140 \mathrm{~g}$. Die Organe werden zerhackt, mit Quarzsand verrieben und mit $1200 \mathrm{ccm}$. destillirten Wassers, dem Toluol im Ueberschuss zugesetzt wird, übergossen. Das Gemisch wird tüchtig durchgeschüttelt und über Nacht auf Eis gestellt. Am nächsten Vormittag $11^{1 / 2}$ Uhr wird der Lebersaft abgehebert, 40 Fläschchen mit je $20 \mathrm{ccm}$. gefüllt, 2 sofort verarbeitet, die übrigen zusammen in denselben Brutschrank gethan, dessen Temperatur meist $38^{\circ}$ betrug, jedoch Schwankungen zwischen 30 und $40^{\circ}$ ausgesetzt war. Täglich wurden dann je 2 Fläschchen verarbeitet.

Die Bestimmung des mit Magnesia austreibbaren Stickstoffs geschah ganz in der Art, wie Hausmann') sie beschrieben hat.

Mit Magnesia austreibbarer Stickstoff wurde gefunden am:

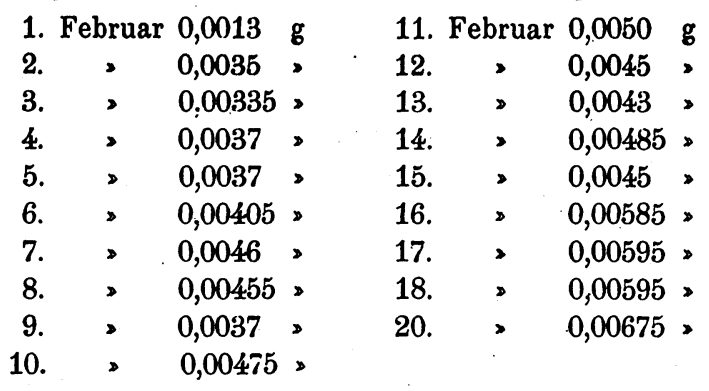

1) Zeitschr. f. physiolog. Chemie, Bd. XXVII. 1899. 
Versuchsprotokoll II. Am 21. Februar 1899 (81/2 Uhr Morgens) wird 2 Hunden die Leber entnommen, ihr Gewicht beträgt $800 \mathrm{~g}$. Sie werden zerhackt, mit Sand zerrieben, mit $800 \mathrm{ccm}$. Toluolwasser extrahirt. Um $10 \mathrm{Uhr}$ Vormittags werden 16 Portionen zu $20 \mathrm{ccm}$. entnommen, von denen 8 sofort mit Magnesia destillirt wurden, die 8 übrigen, nachdem sie bis zum 7. März bei Brutschranktemperatur gehalten worden waren.

Es fand sich an mit Magnesia austreibbarem Stickstoff am:

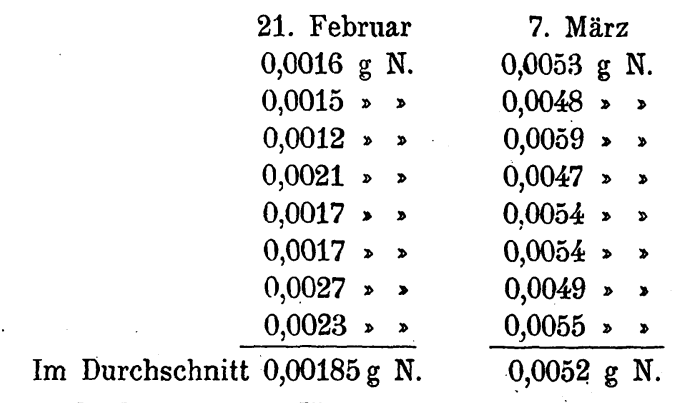

Nach diesen Versuchen, die aus einer Anzahl entsprechender anderer deshalb ausgewählt wurden, weil sie sich auf die grössten Zahlenreihen stützen, ist es als sicher anzusehen, dass der durch Magnesia austreibbare Stickstoff bei der Digestion von Lebersaft zunimmt.

Es lag nahe, diese Zunahme als einen fermentativen Vorgang aufzufassen, und so wurde geprüft, ob nach vorhergehendem Kochen diese Vermehrung des leicht austreibbaren Stickstoffs unterbleibt.

Das ergab sich in der That:

Versuchsprotokoll III. Am 23. Februar 1899 (81/2 Uhr Morgens) wird ein grosser Hund getödtet; die Leber, welche $870 \mathrm{~g}$ wiegt, wird zerhackt, mit Sand zerrieben und mit $800 \mathrm{ccm}$. Toluolwasser extrahirt. Um $9^{1 / 2}$ Uhr werden 12 Fläschchen mit je $20 \mathrm{ccm}$. Lebersaft beschickt, alle Kölbchen aufgekocht, 6 sofort wie in den vorigen Versuchen verarbeitet, 6 bis zum 9. März digerirt.

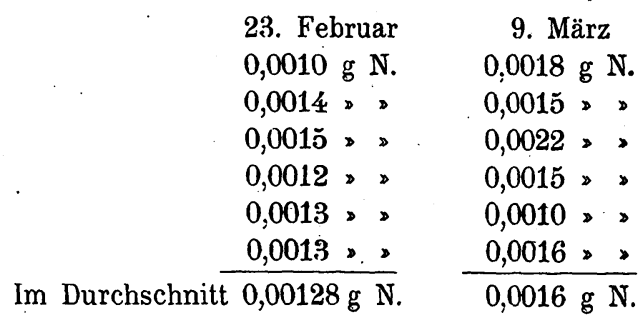


Im gekochten Lebersaft findet also eine merkliche $\mathrm{Ab}$ spaltung von Amidstickstoff nicht statt.

Da Fermente meistens schlecht diffundiren, so deutet auf die Fermentnatur der beobachteten Erscheinung auch nachfolgender Versuch hin, in dem nach der Dialyse des Lebersaftes noch die Bildung des mit Magnesia austreibbaren Stickstoffes nachweisbar war.

Versuchsprotokoll IV. Frischer, mit Toluol versetzter Hundelebersaft wird 40 Stunden gegen fliessendes Wasser dialysirt. Am Schluss der Dialyse ist noch Toluol im Ueberschuss im Schlauch vorhanden. Von dem Schlauchinhalt werden 8 Portionen $\mathrm{zu} 20 \mathrm{ccm}$. abgemessen, zum Theil sofort verarbeitet, zum Theil zur Digestion angesetzt.

Es fand sich Magnesiastickstoff:

\begin{tabular}{cc} 
31. Mai & 13. Juni \\
$0,0003 \mathrm{~g} \mathrm{~N}$. & $0,0016 \mathrm{~g} \mathrm{~N}$. \\
$0,0006 »$ & 0,0019 \\
0,0007 & 0,0017 \\
\cline { 2 - 3 } Im Durchschnitt $0,0005 \mathrm{~g} \mathrm{~N}$. & $0,0017 \mathrm{~g} \mathrm{~N}$.
\end{tabular}

\section{Der durch Magnesia austreibbare Stickstoff entspricht zum grossen Theil neugebildetem Ammoniak.}

Es war nunmehr zu ermitteln, welcher Natur der bei der Digestion entstehende Körper ist, der beim Kochen mit Magnesia Ammoniak abspaltet.

Aus den angeführten Zahlen geht hervor, dass sich entsprechend nachweisbarer Stickstoff bereits in der Leber des eben getödteten Thieres findet. Dass er hier nur in geringer Quantität vorhanden, ist verständlich, wenn man sich überlegt, dass es sich wohl nur um sehr leicht diffusible Substanzen handeln kann, welche in den Organen nicht zur Anhäufung gelangen. Da vermuthlich dieser in der Leber sich vorfindende, mit Magnesia direkt austreibbare Stickstoff verschiedenen Substanzen entstammt, so wäre es schwierig, dieselben im Einzelnen festzustellen, und würde es dazu einer besonderen Untersuchung bedürfen. Für unsere Frage ist dieser Punkt im Augenblick weniger dringlich. Es dürfte daher genügen, nur kurz auf jene Stoffe hinzuweisen, an die zunächst zu denken ist. 
Es ist bekannt, dass sich geringe Ammoniakmengen in der frischen Leber finden; sie müssen in unseren Zahlen mit zum Ausdruck kommen. Ferner ist der Harnstoff betheiligt, der nach Gottlieb ${ }^{1}$ ) sich in der Leber, wenn auch nur in geringer Quantität, nachweisen lässt. ${ }^{2}$ )

Bei der Frage, was für einem Körper der durch Digestion abgespaltene Stickstoff angehört, kommt Harnstoff nicht in Betracht, da ja Loewi unter ähnlichen Verhältnissen die Bildung von Harnstoff vermisste und Gottlieb mit der Oxalsäuremethode auch quantitativ keine Harnstoffzunahme beobachten konnte.

Auch der äther-alkohollösliche Körper Loewi's ist auszuschliessen. Dass es sich um ihn handeln könnte, war von vorneherein unwahrscheinlich, weil bei der Moerner-Sjoequist'schen Methode, mit der Loewi gearbeitet hatte, vor der Aetheralkoholextraction der Theil des Stickstoffs entfernt wird, welcher bei $40^{\circ}$ mit Magnesia zu vertreiben ist. Sicher ausschliessen konnte ich den Loewi'schen Körper deshalb, weil ich bei Versuchen mit Glycocollzusatz keine Mehrbildung von Amidstickstoff nachweisen konnte.

Schliesslich konnte es sich um Ammoniak ${ }^{3}$ ) handeln, was sich auch durch Versuche, in denen die Magnesiamethode und die Schlösing'sche nebeneinander benutzt wurden, sicherstellen liess.

Versuchsprotokoll V. Frischer Lebersaft vom Hund wird am 25. IV. 1899 mit Magnesia destillirt und Kontrollproben 5 Tage nach Schlösing mit Kalkmilch behandelt. Ebenso wird mit einer Anzahl

1) Arch. f. exper. Pathol., Bd. XIII. 1899.

2) Harnstoff zerlegt sich beim Kochen mit Wasser, ob Magnesia zugegen ist oder nicht (vergl. Salaskin und Zaleski, diese Zeitschr., Bd. XXVIII, S. 76). Ich habe bei vielstündigem Kochen von Harnstoff mit Magnesia etwa die Hälfte des Stickstoffs im Destillat nachweisen können; wahrscheinlich lässt sich bei noch längerer Einwirkung von Wasser auf Harnstoff in Gegenwạrt von Magnesia die Spaltung noch weitertreiben. Jedenfalls ist anzunehmen, dass ein Theil unserer kleinen Anfangswerthe vom Harnstoff herstammt.

3) Bezw. um sehr leicht zersetzliche Säureamide. - Wir besitzen zur Zeit keine Methode, um diese von Ammoniak sicher zu trennen. 
Proben am 9. V. 1899 verfahren, nachdem ein Aufenthalt im Brutschrank unter Toluolzusatz vorausgegangen war.

$$
\begin{aligned}
& \text { Magnesiawerthe: } \\
& \text { Schlösingwerthe : } \\
& \text { Vor der Digestion (25. IV.): } \\
& 0,0009 \text { g N } \\
& 0,0013 \text { > } \\
& 0,0013 \text {, } \\
& \text { Durchschnitt } \frac{0,0008 \text {, }}{0,0011 \mathrm{~g} \cdot \mathrm{N}} \\
& 0,0001 \mathrm{~g} \mathrm{~N} \\
& -0,0003 \text {. } \\
& 0,0000 \text {, } \\
& \frac{0,0003 \text { > > }}{0,0000 \text { g N. }} \\
& \text { Nach der Digestion (9. V.): } \\
& 0,0019 \text { g N } \\
& 0,0017 \text { > } \\
& 0,0025 \text {, } \\
& \frac{0,0019 \text { \& }}{0,0020 \mathrm{~g} \mathrm{~N}}
\end{aligned}
$$

Versuchsprotokoll VI wie voriges.

Magnesiawerthe:

Schlösingwerthe:

Vor der Digestion (29. V. 1899):

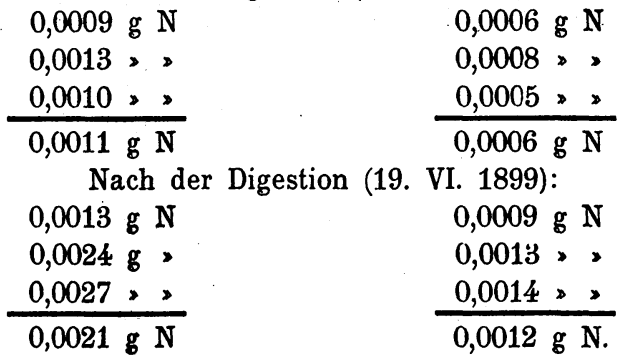

Aus diesen Versuchen geht also hervor, dass Ammoniak bei der Digestion des Lebersaftes entsteht und die Zunahme der Magnesiawerthe mindestens zum Theil darauf zu beziehen ist.

Fermentative Ammoniakbildung innerhalb der thierischen Zellen oder überhaupt das Vorkommen von ammoniakabspaltenden Fermenten in den thierischen Zellsäften ist meines Wissens bisher nicht beschrieben worden, auch über die Verdauungsfermente ist in dieser Richtung nur wenig bekannt. Für das Pepsin hat E. Zunz ${ }^{1}$ ) gezeigt, dass es aus dem Eiweiss Stickstoff abspaltet, der mit Magnesia direkt destillirbar

1) Zeitschr. f. physiol. Chemie, Bd. XXVIII, 1899. 
ist. Ebenfalls mit Magnesia austreibbaren Stickstoff fand schon vor längerer Zeit Hirschler ${ }^{1}$ ) bei der Trypsinverdauung von Fibrin, und Stadelmann ${ }^{2}$ ) konnte zeigen, dass diese Abspaltung keine Fäulnisserscheinung ist.

\section{Aus welchem Material stammt das in der Leber neu gebildete Ammoniak?}

Bereits früher ist erwähnt worden, dass sich auch nach der Entfernung der leicht diffusiblen Substanzen durch Digestion bei Körpertemperatur noch eine Zunahme des Amidstickstoffes erzielen liess.

Da ebensowenig Zusätze von Leberglobulinlösungen wie von Extractivsubstanzen der Leber Ergebnisse lieferten, auch Versuche, das Material zur Ammoniakbildung zusammen mit dem Ferment auszusalzen, nicht zu Resultaten führten, so blieb nur übrig, zu untersuchen, wie sich vor. und nach dem Aufenthalt des antiseptisch behandelten Leberbreies im Brutschrank die Stickstoffvertheilung in demselben gestaltet.

Zunächst gebe ich die Daten einer derartigen Versuchsreihe.

Versuchsprotokoll VII. Am 5. VI. 1899 wird die Leber eines eben getödteten Hundes fein zerhackt, von dem gleichmässigen Brei mehrere Proben von einigen Gramm Gewicht zur Gesammtstickstoffbestimmung entnommen, $100 \mathrm{~g}$ mit Toluolwasser bis zum 23. VI. in den Brutschrank gethan, $100 \mathrm{~g}$ mit stickstoffreiem Zinksulfat unter Hinzufügung von einigen Cubikcentimetern Schwefelsäure gesättigt, im Filtrat der Gesammtstickstoff bestimmt. Da Albumosen in der frischen Leber nicht vorkommen, so konnte die Differenz zwischen Gesammtstickstoff und dem Stickstoff des Zinksulfatfiltrates als Eiweissstickstoff angesehen werden.

Mehrere Proben des Filtrates wurden ferner mit concentrirter Salzsäure 8 Stunden auf dem Sandbade gekocht, dann wurde durch Destillation mit Magnesia der Amidstickstoff bestimmt. Indem ausserdem das Zinksulfatfiltrat direkt mit Magnesia destillirt und so der direkt austreibbare Stickstoff besonders bestimmt wurde, konnte durch Subtraction der durch Säure abspaltbare Amidstickstoff ermittelt werden.

$\mathrm{Da}$ es zu weitläufig sein würde, all die einzelnen, übrigens unter

1) Zeitschr. f. physiol. Chemie, Bd. X, 1886.

2) Zeitschr. f. Biologie, Bd. XXIV, 1888. 
einander gut stimmenden Kontrollanalysen wiederzugeben, so mögen nur die Resultate tabellarisch zusammengestellt werden.

Bemerkt sei noch, dass am Schluss des Versuches mit der digerirten Portion ebenso verfahren wurde. Etwa neu gebildete Albumosen würden hier in die gleiche Fraction wie das Eiweiss fallen, quantitativ ist das jedoch sicherlich unerheblich.

Beginn des Versuches 5. VI. 1899.

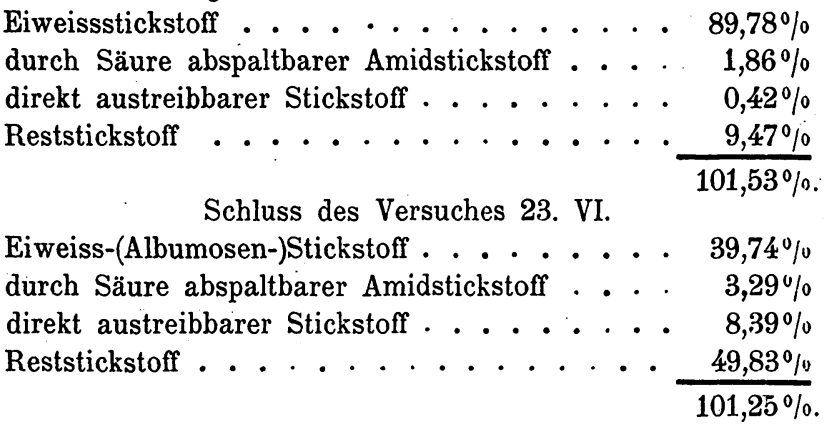

Versuchsprotokoll VIII. In einem anderen Versuche wurde nur der Eiweiss- und der leicht austreibbare Amidstickstoff bestimmt, der Reststickstoff nur berechnet.

Beginn des Versuches 5. I. 1900.

Eiweissstickstoff . . . . . . . . . . . . 9 $94,19 \%$

direkt austreibbarer Amidstickstoff . . . . . 1, 13\%

berechneter Reststickstoff . . . .. . . . . $4,68 \%$

Schluss des Versuches 19. I. 1900.

Eiweiss-(Albumosen-)Stickstoff . . . . . . 27,46\%

direkt austreibbarer Amidstickstoff . . . . . 5, 53\%

berechneter Reststickstoff . . . . . . . 66,91\%

Versuchsprotokoll IX. In einem dritten Versuche wurde ermittelt, dass am Beginn des Versuches am 17. V. 1899 in $100 \mathrm{~g}$ Leber $0,235 \mathrm{~g}$ mit Zinksulfat und Schwefelsäure nicht aussalzbarer Stickstoff vorhanden war, am 30. V. aber in $100 \mathrm{~g}$ Leber 1,656 g.

Bevor wir das Resultat dieser Versuche zusammenfassen, müssen wir bemerken, dass am Schluss der Versuche die Restfraction sicherlich auf eine ganze Reihe von stickstoffhaltigen Substanzen zu beziehen ist, dass aber neben Purinbasen und Diamidosäuren den grössten Ántheil Amidosäuren darstellen. Auffallend ist - worauf später noch zurückgegriffen werden muss -, dass schon bei Beginn der Versuche die Restfraction nicht so ganz unbedeutend ist. Dieser Befund steht nämlich in einem gewissen Widerspruch dazu, dass in frischer Leber qualitativ keine Spur von Amidosäuren nach- 
gewiesen werden konnte. Es ist daher im Auge zu behalten, dass in der frischen Leber diese Fraction im Wesentlichen aus anderen Substanzen bestehen dürfte.

Wie dem auch sei, das unmittelbare Resultat dieser Versuche lässt sich folgendermassen zusammenfassen: Die Eiweisssubstanzen nehmen während des Versuches beträchtlich $a b$, die Restfraction und der leicht austreibbare Stickstoff nimmt gleichzeitig nicht unerheblich zu. Dieser Parallelismus macht es wahrscheinlich, dass die Zunahme speciell des Amidstickstoffes auf Kosten von Eiweiss erfolgt. Doch kann zunächst auf Grund der gefundenen Werthe die Möglichkeit, dass der Ammoniakstickstoff aus dem Stickstoff der Restfraction hervorgeht, nicht ausgeschlossen werden.

Von physiologischen Gesichtspunkten aus ist es jedoch vor Allem wichtig, zu wissen: wird in der Leber Amidstickstoff aus Amidosäurenstickstoff gewissermassen durch eine Lockerung der $\mathrm{NH}_{2}$-Bindung gebildet, und in welchem Umfange? Das kommt für den Stoffwechsel in erster Linie in Betracht, während es in dieser Hinsicht minder wesentlich ist, ob es sich bei der Muttersubstanz um Amidosäuren oder um complexere Bruchstücke des Eiweissmoleküls handelt.

Um diese Frage zu entscheiden, wurde geprüft, ob im Lebersaft bei der Digestion der festgebundene, den Amidosäuren angehörige Stickstoff keine Veränderung erfährt. $\mathrm{Zu}$ dem Zwecke wurde vor und nach Beendigung der Digestion eine Bestimmung des durch Säure nicht abspaltbaren Stickstoffs vorgenommen. Die Eiweisskörper wurden, der Fragestellung dieser Versuche entsprechend, natürlich nicht entfernt.

Es ergab sich Folgendes:

Versuchsprotokoll X.

In dem Versuch betrug in $\%$ des Gesammtstickstoffs ausgedrückt:

\begin{tabular}{|c|c|c|}
\hline . & $\begin{array}{c}\text { Am Beginn } \\
\text { der } \\
\text { Digestion }\end{array}$ & $\begin{array}{c}\text { Am Schluss } \\
\text { der } \\
\text { Digestion }\end{array}$ \\
\hline Der durch Säure abspaltbare Amidstickstoff . & $4,7 \%$ & $9,3 \%$ \\
\hline Der direkt austreibbare Amidstickstoff & $4,9 \%$ & $6,2 \%$ \\
\hline Der gesammte Amidstickstoff . & $9,6 \%$ & $15,5 \%$ \\
\hline
\end{tabular}




\begin{tabular}{lr|r}
\hline \hline & $\begin{array}{c}\text { Am Beginn } \\
\text { der } \\
\text { Digestion }\end{array}$ & $\begin{array}{c}\text { Am Schluss } \\
\text { der } \\
\text { Digestion }\end{array}$ \\
\hline Der durch Säure abspaltbare Amidstickstoff . . . & $8,7 \%$ & $6,3 \%$ \\
Der direkt austreibbare Amidstickstoff . . . - & $2.6 \%$ & $9,4 \%$ \\
\hline Der gesammte Amidstickstoff . - $11,3 \%$ & $15,7 \%$
\end{tabular}

Der gesammte Amidstickstoff hat also in beiden Versuchen deutlich zugenommen, es geht also fest gebundener Stickstoff in Amidstickstoff über. Dieses Resultat steht mit der Beobachtung Loewi's in Einklang, dass Glycocoll sich bei der Digestion mit Leberfermentlösung in einen Körper umwandelt, der leicht abspaltbaren Stickstoff aufweist.

\section{Beziehung der beschriebenen fermentativen Vorgänge zu Salko'wski's Autodigestion.}

Die im vorigen Kapitel wiedergegebenen Versuche haben gezeigt, dass zwischen der Abnahme der Eiweisskörper und der Abspaltung von Ammoniak in der Leber ein ausgesprochener zeitlicher Parallelismus besteht, und dass die Ammoniakbildung auch mit dem von Loewi studirten Process in Beziehung zu bringen ist. Indem ich zunächst auf eine weitere Prüfung der Frage, ob wir es hier mit einer einheitlichen Fermentwirkung $\mathrm{zu}$ thun haben, verzichtete, ging ich daran, zu untersuchen, ob diese Vorgänge mit der von Salkowski entdeckten und von ihm und seinen Schülern studirten Autodigestion in Zusammenhang stehen.

Die Arbeit Salkowki's, die anscheinend trotz ihrer grossen Bedeutung nicht hinreichende Beachtung in der Litteratur gefunden hat, bildete den Ausgangspunkt für unsere jetzt zu besprechenden Versuche.

Man gewinnt bei einem Ueberblick über die bei der Autodigestion gegebenen Vorgänge den Eindruck, dass wir hier eine fundamental wichtige Einrichtung des Organismus vor uns haben, eine Einrichtung, welche mit Hülfe fermentativer Umsetzung den Abbau des Zelleiweisses in den Organen in der Weise ermöglicht, dass aus geformtem Zellmaterial lös- 
liches, aus nicht diffusiblen Bestandtheilen diffusible und daher leichter aus den Organen zu eliminirende Produkte gebildet werden.

Im Hinblick auf diese Auffassung möchte ich vorschlagen, Vorgänge dieser Art, die sich nicht nur bei postmortaler Digestion, sondern, wie ich Gelegenheit haben werde, zu zeigen, auch im lebenden Organismus vollziehen und beim normalen Abbau der Gewebe eine massgebende Rolle spielen dürften, als autolytische zu bezeichnen, wobei die Analogie mit anderen, zum Theil direkt hierher gehörigen Vorgängen, wie Karyolyse, Hämolyse u. s. w. deutlicher hervortritt als bei Verwendung der von Salkowski vorläufig gewählten Bezeichnung «Autodigestion», bei welcher man unwillkürlich hauptsächlich an die experimentelle Digestion extra corpus denkt.

Zunächst mögen in Kürze die einschlägigen Befunde von Salkowski, Schwiening und Biondi wiedergegeben werden.

Salkowski1) fand, dass mit Chloroformwasser digerirter Leberbrei Veränderungen der Art erleidet, dass organische und insbesondere auch stickstoffhaltige Substanz in Lösung geht. Da vor der Digestion gekochte Leber diese Erscheinung nicht zeigt, ist anzunehmen, dass es sich um eine Fermentwirkung handelt. Bei dieser Selbstverdauung der Leber sah Salkowski Leucin und Tyrosin auftreten. Ausserdem werden in der Arbeit ähnliche Beobachtungen über Hefe- und Muskelautodigestion, sowie über das Verhalten der Nucleine mitgetheilt, auf die in dieser Arbeit nicht eingegangen werden braucht.

Schwiening ${ }^{2}$ ) stellte dann fest, dass mikroskopisch zellfreie Filtrate dieselben Veränderungen erleiden, wodurch die Enzymnatur des Vorgangs noch mehr sichergestellt wurde, ausserdem studirte Schwiening den Einfluss des Alkali auf die Autolyse.

Aus der Arbeit von Biondi, ${ }^{3}$ ) der nach verschiedenen Richtungen die Arbeiten Salkowski's und Schwiening's

1) Zeitschr. f. klin. Med. 1891. Suppl.

2) Virchow's Archiv. 1894.

3) Virchow's Archiv. 1896. 
weiterführte, sei hier nur Einiges hervorgehoben. Biondi zeigte, dass man ausser den Organen der von Salkowski benutzten Versuchsthiere (Hund und Kaninchen) auch jene anderer Thiere, z. B. Kalbsleber, zu den Experimenten über Autodigestion benutzen kann. Ferner konnte auch Autolyse nachgewiesen werden, wenn man an Stelle von Chloroform andere Antiseptica anwandte, so dass der Einwand, es handle sich um Chloroformwirkung, nicht erhoben werden kann. Endlich stellte Biondi vergleichende Untersuchungen über die Wirkung der Autodigestion der Leber und die Einwirkung von Trypsin auf Leber an und fand neben quantitativen Unterschieden, auf die er mit Recht wenig Werth legt, auch qualitative Verschiedenheiten. Als wesentlichsten Unterschied sieht er an, dass bei der Autodigestion in seinen Beobachtungen kein Tryptophan gebildet wurde, während Tryptophanbildung ein regelmässiger Befund bei der Pankreasverdauung ist.

So viel auch, wie ich weiter unten zeigen kann, für die Anschauung Salkowski's, die Biondi mit dieser Beobachtung gegen Neumeister stützen wollte, spricht, dass nämlich die Leberzellen ein eigenes, eiweissspaltendes Ferment bilden, so müssen wir doch unten Beobachtungen mittheilen, welche darthun, dass hier in Bezug auf die Bildung von Tryptophan auch nur quantitative resp. zeitliche Differenzen gegenüber dem Trypsin bestehen.

\section{Ueber die bei der Autolyse der Leber auftretenden Produkte.}

Wenn nun im Folgenden einige eigene Beobachtungen mitgetheilt werden sollen, so werde ich nicht über sämmtliche in dieser Richtung angestellten Versuche ausführlich berichten, da Vieles - wenn auch mit anderen Methoden gewonnen lediglich eine Bestätigung der Angaben Salkowski's und seiner Schüler brachte. Ich werde nur das herausgreifen, was als Ergänzung oder Weiterführung der bisherigen Kenntnisse angesehen werden kann.

Salkowski hat bereits hervorgehoben, dass anscheinend ausser Leucin und Tyrosin und den aus den Nucleinen stammenden Purinbasen bei der Autolyse noch andere 
stickstoffhaltige Produkte gebildet werden. Dafür sprechen die quantitativen Versuche.

Albumosen werden - wenn überhaupt - sicher nur in geringer Quantität gebildet, wie ich mich in Bestätigung der früheren Angaben überzeugt habe. Auch scheinen sie nur ganz vorübergehend vorhanden zu sein, da sie in älteren Digestionsflüssigkeiten in mehreren Versuchsreihen ganz vermisst wurden.

Peptone wurden, wie von Salkowski etc., auch von mir nicht gefunden.

In Bezug auf die tiefer stehenden Produkte wäre es Aufgabe einer systematischen Untersuchung, nach all' den Substanzen zu fahnden, welche bei der Einwirkung anderer spaltender Agentien und namentlich der Verdauungsfermente aufgefunden worden sind. Im Rahmen dieser Arbeit konnten derartige Versuche nur im kleinsten Umfange angestellt werden.

Bereits ausführlich mitgetheilt sind die Beobachtungen über die Bildung von Ammoniak und Amidstickstoff bei der Autolyse (siehe voriges Kapitel). Hier konnte auch der quantitative Antheil festgestellt werden.

Ferner werden basische Produkte gebildet. Man erhält nach vollkommener Entfernung der Eiweisskörper, einschliesslich etwa gebildeter Albumosen, mit Phosphorwolframsäure sehr massige Niederschläge, in der frischen Leber nur geringfügige. Auch nach Entfernung des Ammoniaks mit Magnesia ist noch mit Phosphorwolframsäure fällbare Substanz nachweisbar. $\mathrm{Ob}$ es sich dabei nur um die Bildung von Purinkörpern handelt oder auch anderer Basen, bleibt noch zu untersuchen. Mit Benzoylchlorid und Aetznatron erhält man reichliche Massen eines bei alkalischer Reaction unlöslichen Niederschlages. Derselbe ist in Aethyl- und Methylalkohol und in Chloroform löslich, kann mit Wasser und mit Ligroin aus den Lösungen gefällt werden. Die Benzoylverbindung ist stickstoffhaltig, liefert bei der Kalischmelze kein Skatol und geht leicht in eine schmierige Masse über.

Von Amidosäuren wurde neben relativ grossen Quan- 
titäten von Leucin und Tyrosin auch Glycocoll mit an Sicherheit grenzender Wahrscheinlichkeit nachgewiesen.

Versuchsprotokoll XII. Die Verdauungsflüssigkeit wurde zunächst durch Filtriren von den festen Bestandtheilen befreit, dann eingeengt. Grosse Quantitäten Tyrosin werden durch Abfiltriren entfernt, dann wird das Filtrat mit Benzoylchlorid und Aetznatron tüchtig geschüttelt, die bei alkalischer Reaction ausfallenden Massen werden abfiltrirt, das Filtrat wird angesäuert und mehrfach mit Essigäther und Aether extrahirt. Mit Ligroin wird die Benzoesäure entfernt, wobei die nicht in das Ligroin übergehende Substanz deutlich ausgefällt wird. Endlich wird die ätherlösliche Fraction mit Benzaldehyd unter Hinzufügung von Natriumacetat und Essigsäureanhydrid nach den Angaben von Erlenmeyer jun.1) und Spiro2) condensirt. Man erhält schöne, seidenglänzende Nadeln. Dieselben werden aus heissem Alkohol, in dem sie löslich sind, zwei Mal umkrystallisirt. Die Krystalle schmelzen bei $160^{\circ}$, während der Schmelzpunkt des Lactimids, das Erlenmeyer jun. als Condensationsprodukt aus Hippursäure und Benzaldehyd erhalten hat, bei $165-166^{\circ}$ schmilzt. Weiteres Umkrystallisiren, das wohl den Schmelzpunkt in die Höhe gebracht hätte, wurde durch einen zufälligen Verlust des Materials verhindert.

Dieser Befund des charakteristischen Lactimids zeigt an, dass Hippursäure in der Lösung vorhanden war. Da von mir niemals Amidosäuren in der frischen Leber gefunden wurden, Hippursäure in der Rindsleber nicht nachgewiesen worden ist, auch Glycocholsäure, falls Spuren vorhanden sein sollten, durch das angewandte Verfahren nicht würde gespalten werden, so ist es das Naheliegendste, das gefundene Glycocoll als autolytisches Produkt der Leber aufzufassen.

Das Vorkommen von Glycocoll unter den Digestionsprodukten der Leber dürfte physiologisches Interesse haben. Seitdem Spiro gezeigt hat, dass Glycocoll ein Spaltungsprodukt einer grösseren Anzahl von Eiweisskörpern ist, erscheint es nicht überraschend, wenn bei der Autolyse der Leber neben Leucin und Tyrosin auch Glycocoll auftritt. Da ferner die Glycocholsäure in der Leber des lebenden Thieres gebildet wird, liegt die Vermuthung nahe, dass auch das an Cholsäure gebundene Glycocoll ein Produkt des autolytischen Processes ist.

Ferner ist es namentlich nach den Untersuchungen von Wiener ${ }^{3}$ )

1) Berichte der chem. Gesellsch. Bd. $25-30$.

2) Zeitschr. f. physiol. Chemie. Bd. XXVIII, 1899.

3) Arch. f. exper. Pathol., Bd. XXXX u. XXXXII, 1898 u. 1899. 
und Weiss 1) wahrscheinlich, dass Glycocoll aus Harnsäure, und Harnsäure aus Glycocoll im Organismus gebildet werden kann; da nun ebenfalls nach Wiener in der Rindsleber eine gleichzeitige Entstehung und Zerstörung von Harnsäure angenommen werden darf, so ist es möglich, dass Glycocoll in der Leber Vorstufe wie Abkömmling der Harnsäure ist.

Schliesslich sei noch daran erinnert, dass Loewi das Glycocoll durch das Richet'sche Leberferment in einen äther-alkohollöslichen, stickstoffhaltigen Körper überführen konnte, und dass Glycocoll sehr vollkommen im Organismus in Harnstoff umgewandelt wird.

Bei mehreren Versuchen sah ich als einen Hinweis auf ein weiteres Produkt während der Autolyse die Tryptophanreaction auftreten. Während die frische Leber mit Bromwasser keine Reaction gab, erhielt man nach ca. 14 tägiger Selbstverdauung mit Bromwasser eine starke Violettfärbung. In anderen Fällen wurde keine Bromreaction beobachtet. Auch Biondi hat sie nur dann gefunden, wenn sie schon in der frischen Leber vorhanden war. Wahrscheinlich tritt sie erst spät und auch dann nur vorübergehend auf. Ihr Fehlen kann jedenfalls nicht als Unterscheidungsmerkmal der Leberautolyse gegenüber der Trypsinwirkung gedeutet werden.

Soweit es gestattet ist, die mit Leberauszïgen erhaltenen proteolytischen Vorgänge mit Trypsinwirkung zu vergleichen, so ergeben sich als unterscheidende Momente:

1. Der Verlauf der autolytischen Spaltung ist ein langsamer, trozdem tritt die Bildung von Albumosen dabei zurück, während die Bildung von Endprodukten überwiegt.

2. Dabei erfolgt eine Ueberführung von fest gebundenem Stickstoff in locker gebundenen, was bei der Trypsinverdauung nicht der Fall oder wenigstens bisher nicht bemerkt ist.

3. Das proteolytische Ferment der Leber wirkt auslesend, nicht auf alle, sondern nur auf bestimmte Eiweissstoffe ein (s. nächstes Kapitel).

Das Bestehen dieser Differenzen würde ganz gut damit im Einklang stehen, dass beiden Fermenten im Organismus

1) Zeitschr. f. physiol. Chemie, Bd. XXV, 1898. 
auch verschiedene Functionen zufallen (dem Trypsin Vorbereitung der Nahrungsstoffe für die Resorption und Assimilation, der Autolyse der Abbau des Organeiweisses und Vorbereitung zur Harnstoffbildung). Man darf jedoch auch nicht ausser Acht lassen, dass im Leberauszug mehrere Fermente vorhanden sind, und dass möglicher Weise die beobachteten Wirkungen erst durch ihr Zusammenwirken zu Stande kommen, wobei dann eine Uebereinstimmung mit der isolirten Trypsinwirkung nicht $\mathrm{zu}$ erwarten ist.

Vorläufig ist jedenfalls die Identität des Trypsins und des Ferments derLeberautolyse nicht bewiesen, und es liegt kein ausreichender Grund vor, eine derartige Identificirung vorzunehmen.

Anhangsweise seien einige ursprünglich aus anderen Gründen ausgeführte Versuche über Autolyse der Vogelleber erwähnt.

Chassevant und Richet haben in der Annahme, dass a priori ein sferment uropoïétique in der Vogelleber nicht zu erwarten wäre, geprüft, ob die Vogelleber ein harnstoffbildendes Ferment besitzt. Sie verneinen es, da sie keine Abnahme zugesetzter Harnsäure feststellen konnten. 1) (Compt. rend. de la société de biol., 1898, Nr. 32.)

Ich habe gefunden, dass die Gänseleber bei der Autolyse Leucin und Tyrosin und daneben basische Produkte liefert, deren Natur aus Mangel an Material nicht ermittelt werden konnte.

\section{Welche Eiweissstoffe unterliegen der autolytischen Spaltung?}

Da die gefundenen Produkte der Autolyse sich als Spaltungsprodukte von Proteinstoffen herausstellen, so ist zunächst die Frage zu prüfen, welche Eiweisskörper der Leber durch das autolytische Ferment vorzugsweise angegriffen werden.

$\mathrm{Zu}$ diesem $\mathrm{Zwecke}$ habe ich nachstehenden Versuch ausgeführt:

Versuchs-Protokoll XIII. Eine grössere Quantität Leberbrei wird mehrere Monate mit Toluolwasser bei Brutschranktemperatur sich selbst überlassen.IN Nach einigen Wochen wird der ungelöste Rückstand durch Filtriren fentfernt, wiederum nach einer Woche die Hauptmasse der diffusiblen Produkte durch Dialyse gegen fliessendes Wasser beseitigt

1) Es mag hier übrigens?̨̣uf ein Missverständniss der Autoren hingewiesen werden. Sie schreiben, Loewi habe das Vorkommen eines harnstoffbildenden Ferments in der Leber der Säuger bestätigt; es ist dafür zu setzen : «nicht bestätigt». 
und die Verdauung im Brutschrank fortgesetzt, nachdem durch Kochsalzzusatz ein Salzgehalt von etwa 1\% hergestellt war. Während der Dialyse bildet sich in der Flüssigkeit ein Niederschlag, der auch nicht nach dem Kochsalzzusatz verschwindet, sondern bei der fortgesetzten Digestion noch zunimmt. Schliesslich wird nach drei Monaten festgestellt, dass die Eiweissspaltung ihren Abschluss gefunden hat.

Sodann wird der Niederschlag durch Filtriren von der Lösung getrennt. Er ist von dunkler Farbe, in Wasser ganz unlöslich. Derselbe wird mit viel Wasser gewaschen; er ist in der Kälte weder in Natronlauge, noch in Essigsäure, auch bei starker Concentration der Lösungsmittel löslich. Beim Kochen mit Natronlauge löst sich der Niederschlag, kann dann mit Essigsäure wieder ausgefällt werden und ist nunmehr im Ueberschuss von Essigsäure löslich. Beim Kochen mit Essigsäure bleibt er ungelöst.

Die Substanz enthält eine nicht únbeträchtliche Menge Phosphor.

Die Xanthoproteinreaction ist deutlich positiv.

Die Molisch'sche Probe, die Reaction von Tollens mit Phloroglucin und Salzsäure und die Millon'sche Reaction fallen negativ aus.

Mit Pepsinsalzsäure wird eine Probe in 24 Stunden bis auf einen geringen Rest verdaut. Der Rest sowohl, wie auch die in Lösung gegangene Portion ist phosphorhaltig.

Neben diesem unlöslichen Körper, der wahrscheinlich ein Gemisch von Nucleinen mit einem Gobulin und vielleicht noch mehr Substanzen darstellt, findet sich in der Lösung ein coagulabler Eiweisskörper. Derselbe wird durch Ammonsulfat erst bei einer Sättigung von über $60 \%$ ausgesalzen, die Coagulation durch Hitze beginnt bei $75^{\circ}$, während bei $80^{\circ}$ deutlich Flocken gebildet werden. Der mehrfach durch Aussalzung und wiederholtes Lösen gereinigte Körper gibt eine deutliche Reaction mit Millon's Reagens, eine schwache Xanthoproteinreaction, keine Spur von Reaction mit $\alpha$-Naphtol und Schwefelsäure. Der lösliche Eiweisskörper zeigt somit im Ganzen das Verhalten eines Albumins.

Albumosen werden nicht gefunden.

Das Auffallendste dieses Resultates dürfte sein, dass sich keine Spur von Globulin mehr in der Lösung befand, während sich in einem nicht verdauten Leberauszug, auch nach starker Dialyse und auch ohne neuen Salzzusatz, immer noch reichlich ein Eiweisskörper nachweisen lässt, der sich in Bezug auf Aussalzbarkeit wie ein Globulin verhält.

\section{Versuche zur Isolirung des bei der Autolyse wirksamen proteolytischen Fermentes.}

Wenn wir die Wirkungen der Autolyse ins Auge fassen, so sehen wir durch sie Spaltung von Eiweiss, daneben Bil- 
dung von Amidosäuren und Ammoniak erfolgen. Sicherlich sind diese Leistungen von physiologischem Interesse, da der beim Absterben von Zellindividuen oder Zelltheilen in der Norm erfolgende Abbau der Eiweisskörper und die Bildung wichtiger intermediärer Produkte auf die Wirkung von Organfermenten zurückgeführt erscheint. Genauere Studien über die Wirkungen der dabei thätigen Fermente werden aber erst möglich sein, wenn ihre Isolirung einigermassen gelungen sein wird.

Im Folgenden soll über solche Isolirungsversuche berichtet werden.

Nach Analogie anderer Fermente war anzunehmen, dass das autolytische Leberferment aussalzbar sein würde.

Die Leber wurde daher zunächst mit Toluolwasser der Autolyse überlassen. Hierbei gingen, wie in den früher wiedergegebenen Versuchen, grosse Quantitäten aussalzbarer Substanzen in nicht aussalzbare über, und man befreite so das Ferment von vorne herein von grossen Massen anhaftender Substanz.

In der That wurde dann erst oberhalb einer 60\%igen Sättigung mit Ammonsulfat: in dem 14 Tage digerirten Leberauszug ein Niederschlag erhalten. Nachdem Salz bis zur Sättigung zugefügt worden war, setzte sich allmählich ein reichlicher Niederschlag ab, der nach dem Abfiltriren in Wasser gelöst wurde.

Diese Lösung war klar und wirkte deutlich verdauend auf Leberbrei.

Versuchsprotokoll XIV. Von der Lösung werden 2 Portionen à $50 \mathrm{ccm}$., vơn denen die eine vorher gekocht wird, mit je $100 \mathrm{~g}$ frischer Hundeleber und $30 \mathrm{ccm}$. Wasser und einer reichlichen Quantität Toluol zusammengebracht und 16 Stunden bei Brutschranktemperatur gehalten. Dann wird in beiden Kontrollversuchen sorgfältig enteiweisst und in den Filtraten der nicht coagulable Stickstoff bestimmt.

Leber + gekochtes Ferment ergab 0,688 nicht coagulablen Stickstoff

. + ungekochtes $\gg 1,124$,

1) In Uebereinstimmung mit den im vorigen Kapitel mitgetheilten Versuchen über die Betheiligung der einzelnen Lebereiweisskörper an der Autolyse. 
Versuchsprotokoll XV. Die Leber eines eben getödteten Hundes wird zerhackt. Der Brei wird mit $25 \mathrm{ccm}$. 1/10 Normalschwefelsäure durchmischt und 2 Stunden so belassen. Dann neutralisirt man die Säure durch eine entsprechende Quantität Normalnatronlauge und wägt von dem so behandelten Brei zwei Portionen à $200 \mathrm{~g} \mathrm{ab}$. Zu beiden werden je $50 \mathrm{ccm}$. Fermentlösung (wiederum die eine Probe vorher gekocht) hinzugethan und nun wird wie im vorigen Versuche verfahren mit dem Unterschiede, dass der Aufenthalt im Brutschrank auf 3 Tage ausgedehnt wird.

Es wurde nicht coagulabler Stickstoff gefunden:

Leber + gekochtes Ferment 2,542 g

$\checkmark+$ ungekochtes > 3,604

Bei vollkommener Sättigung mit Ammonsulfat wird also ein Ferment ausgezalzen, das deutlich auf Leberbrei verdauend einwirkt. Bei $80 \%$ Sättigung mit Ammonsulfat wurde in einem anderen Versuche nichts erhalten. Das Ferment scheint also erst bei vollständiger Sättigung mit Ammonsulfat ausgesalzen $\mathrm{zu}$ werden.

Diese Feststellung beansprucht von verschiedenen Gesichtspunkten aus Interesse. Zunächst wird es nunmehr nicht unmöglich sein, in der Isolirung des Fermentes weiter zu kommen. Ferner ist hervorzuheben, dass dieses Verhalten durchaus mit dem Verhalten des in der Wirkung so ähnlichen Pankreastrypsins übereinstimmt. Endlich ermöglicht uns das Aussalzungsverfahren eine scharfe Trennung dieses Lebertrypsins von der Aldehydase der Leber, was für physiologische Versuche von Wichtigkeit ist.

$\mathrm{Zu}$ dem letzten Punkte sei hier gleich bemerkt, worauf in einer folgenden Mittheilung in anderem Zusammenhange noch wird eingegangen werden, dass die Aldehydase durch Autolyse nicht zerstört wird; in Leberauszügen, welche längere Zeit digerirt waren, wurde sie noch deutlich wirksam angetroffen.

\section{Wirkung der durch Autolyse erhaltenen Lösungen auf Hippur- säure, Harnstoff und Blutgerinnung.}

Neben der Isolirung des Fermentes ist es für ein specielleres Studium der proteolytischen Leberfunction nöthig, möglichst einfach constituirte Substanzen aufzufinden, welche von Leberauszügen hydrolytisch gespalten werden und event. als Reagentien zum Nachweis autolytischer Fermente dienen 
könnten. Es wurden Versuche mit Hippursäure und Harnstoff angestellt, die hier ihre Stelle finden sollen, weil die Leberextracte aus ihnen Produkte abspalten (Glycocoll resp. Ammoniak), die wir als Produkte der Autolyse kennen gelernt haben. Natürlich können wir vorläufig nur aussagen, dass Leberauszüge diese Substanzen spalten - ob das eine Leistung des proteolytischen Fermentes darstellt, muss zunächst unentschieden bleiben.

Versuchsprotokoll XVI. Rindslebersaft, der bereits mehrere Wochen unter Toluolzusatz der Selbstverdauung ausgesetzt war, wird mit einem Tropfen dünner Sodalösung schwach alkalisch gemacht, dann mit benzoesäurefreier Hippursäure versetzt. Nach viertägigem Aufenthalt im Brutșchrank wird durch Ausschüttelung mit Ligroin Benzoesäure aus der Lösung isolirt, deren Schmelzpunkt bei $119,5^{\circ}$ liegt, während reine Benzoesäure bei $121^{\circ}$ schmilzt.

Versuchsprotokoll XVII. In einem zweiten gleich angestellen Versuche wird ebenfalls Benzoesäure gefunden.

Schmiedeberg ${ }^{1}$ ) hat bereits vor längerer Zeit gefunden, dass die Leber Hippursäure spaltet, indem er sein Hippursäure spaltendes «Histozym» unter Cautelen, die Fäulnisseinwirkungen unwahrscheinlich machten, in der Leber nachwies. - Minkowski²) fand das Schmiedeberg'sche Histozym nicht in der Leber.

Nach Nencki ${ }^{3}$ ) wird vom Pankreas Hippursäure gespalten, Gulewits $\mathrm{ch}^{4}$ ) beobachtete bei Versuchen mit Trypsin, das völlig vom fettspaltenden Pankreasferment befreit war, keine Hippursäurespaltung. - Da auch für die Leber eine lipolytische Function nachgewiesen ist, so muss die Frage, welches Ferment der Leber bei der Hippursäurespaltung betheiligt ist, noch genauer untersucht werden.

Die Spaltung von Harnstoff durch Lebersaft unter Bildung von Ammoniak lehrt folgender Versuch.

Versuchsprotokoll XVIII. Rindslebersaft, der einige Zeit mit Toluol aufgehoben war, wird in Portionen von je $40 \mathrm{ccm}$. getheilt. Sodann werden folgende Kontrolllösungen hergestellt:

1) Arch. f. exper. Path., Bd. XIV, 1881.

2) Arch. f. exper. Path., Bd. XVII, 1883.

3) Arch. f. exper. Path., Bd. XX, 1886 (nach Versuchen von Blank).

4) Zeitschr. f. physiolog. Chemie, Bd. XXVII. 1899. 
$\left.\begin{array}{l}\text { 1. u. 2. } 40 \mathrm{ccm} \text {. Leberauszug } \\ 40 \gg \text { Aq. dest. }\end{array}\right\}$ Toluol

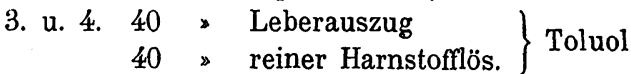

$\left.\begin{array}{l}5 . \quad 20 \gg \text { derHarnstofflös. } \\ 40 \gg \text { Aq. dest. }\end{array}\right\}$ Toluol

$\left.\begin{array}{rl}40 & \gg \text { der Harnstofflös. } \\ 40 & \text { Aq. dest. }\end{array}\right\}$ Toluol

Die Kölbchen mit den Lösungen werden 36 Stunden bei Brutschranktemperatur gehalten. Danach kommen alle Proben auf 5 Tage in Schlösing'sche Apparate.

Es wird Ammoniak-Stickstoff gefunden:

I. Leberauszug allein: $0,0044 \mathrm{~g}$

II. Leberauszug $+40 \mathrm{ccm}$. Harnstofflösung $0,0097 \mathrm{~g}$

III. $40 \mathrm{ccm}$. Harnstofflösung $0,0002 \mathrm{~g}$.

Der Lebersaft hat also aus Harnstoff $0,0051 \mathrm{~g}$ AmmoniakStickstoff gebildet. ${ }^{1}$ )

Auf dieses Resultat komme ich in einer späteren Mittheilung über die Harnstoffbildung in der Leber noch zurück; hier soll nur betont werden, dass wir danach im Harnstoff einen Körper besitzen, den wir gleichsam als Indicator bei weiteren Versuchen über Autolyse der Leber benutzen können.

Die Beziehungen, welche in neuerer Zeit zwischen der gerinnungshemmenden Wirkung der Albumosen und der Leberfunction ${ }^{2}$ ) gefunden worden sind, veranlassten mich, den Einfluss der autolytischen Auszüge auf die Blutgerinnung zu untersuchen.

Injicirte ich nicht zu kleine Quantitäten durch Autolyse erhaltenen, proteolytisch wirksamen Lebersaftes in die Vena femoralis eines Hundes, so veränderte sich durchaus nicht

1) Anm. bei der Correctur: Ein Versuch, bei dem die Ammoniakbestimmungen nach der Methode von Nencki-Zaleski ausgeführt wurden, bestätigte dieses Resultat; er zeigte, dass gekochter Lebersaft Harnstoff nicht spaltet und ergab endlich, dass 17,4\% des HarnstoffStickstoffs in Ammoniakstickstoff übergeführt wurden.

2) Litteratur s. bei Spiro u. Ellinger, Zeitschr. f. physiol. Chemie, Bd. XXIII, 1897, welche wohl am einwandsfreiesten bewiesen haben, dass Witte-Pepton nicht direkt das Blut ungerinnbar macht, sondern erst nach Passage der Leber. - Eine sehr vollständige Litteraturübersicht gibt auch Arthus, La coagulation du sang. 
die Blutgerinnbarkeit, während das Thier auf Einspritzung von Witte-Pepton prompt reagirte.

Positive Resultate gaben dagegen Versuche, bei denen direkte Mischung von Lebersaft mit Blut vorgenommen wurde.

Versuchsprotokoll XIX. Lebersaft, der durch 36 stündige Dialyse gegen fliessendes Wasser von der Hauptmasse der diffusiblen Produkte befreit und nachher auf $1 \%$ igen Kochsalzgehalt gebracht war, wird mit Blut eines normalen Hundes zusammengebracht. Das Gemisch gerinnt sofort. Nach etwa $1 / 4$ Stunde aber beginnt sich das Gerinnsel bereits zu lösen, und diese *Fibrinolyse» schreitet allmählich immer weiter.

Versuchsprotokoll XX. Ein anderer Lebersaft, aus dem die Produkte der Autolyse nicht durch Dialyse entfernt waren, verhindert etwa $1 / 2$ Stunde die Gerinnung zugesetzten Hundeblutes schon in kleineren Quantitäten. Dann tritt Gerinnung ohne nachträgliche Fibrinolyse ein. Derselbe Lebersaft, nur vorher gekocht, verhält sich ebenso.

Ich verzichte auf eine Discussion dieser vereinzelten Befunde, die eine Fortsetzung der Versuche wünschenswerth erscheinen lassen.

IX. Findet die autolytische Spaltung auch im lebenden Gewebe statt?

Schliesslich wurde geprüft, ob sich Bedingungen schaffen lassen, unter denen man bereits intra vitam die Autolyse der Leber beobachten könnte.

Die Versuche wurden nach zwei Methoden ausgeführt. ${ }^{1}$ )

Hunden wurde die Arteria hepatica und die Vena portae unterbunden, nach einigen Stunden die Ligatur gelöst. Ein Thier ${ }^{2}$ ) überlebte die Operation eine Anzahl Stunden, und in seiner Leber fand sich Leucin und Tyrosin.

Bei anderen Hunden wurde ein Theil der Leber dauernd unterbunden. Diese Thiere lebten etwas länger. Auch hier konnte bei einem Hunde, der 36 Stunden die Operation überlebt hatte, im abgebundenen Lappen Leucin und Tyrosin nachgewiesen werden, in den normalen Theilen der Leber nicht.

Da ich aber zur Zeit noch von keinem Hund, der die

1) Dieselbe Frage wurde auch mit Hülfe der Phosphorvergiftung studirt. - Ueber diese Versuche berichte ich in der folgenden Mittheilung.

2) Die meisten Thiere starben bei der Lösung der Ligatur. 
Operation lange genug überlebt hat, und bei dem ein hinreichend grosser Leberlappen abgebunden war, eine bakteriologische Untersuchung mit negativem Befund besitze, so müsste man, trotzdem immer nur im abgebundenen Gewebe die Produkte gefunden wurden, während bei den bakterienhaltigen Organen die Bakterien stets auch im normalen Gewebe gefunden wurden, ${ }^{1}$ ) die Frage noch als unerledigt ansehen.

Nun habe ich aber ausserdem nach der Methode, die ursprünglich Meissner angegeben hat und mit der später Hauser und Fr. Kraus gearbeitet haben, einen Versuch mit völlig aseptischen Leberstücken ausgeführt, der zu einem unzweideutigen, positiven Resultat führte.

Diesen Versuch werde ich daher etwas genauer schildern.

Versuchsprotokoll XXI. Unter ähnlichen Cautelen, wie $\mathrm{Kraus}^{2}$ ) sie angewandt und beschrieben hat, werden einem Hunde, der vorher mit Chloroform getödtet wird, Leberstücke von ca. $20 \mathrm{~g}$ entnommen und möglichst schnell in vorher sterilisirte, mit Wattebausch verschlossene Glasgefässe gebracht. So werden sie bei Brutschranktemperatur längere Zeit belassen. Bereits am nächsten Morgen sind auffallende Erscheinungen zu constatiren. Die Farbe der Leber ist bereits verändert, die Röhrchen lassen einen eigenthümlichen Geruch, ähnlich dem von zersetztem Eiweiss, erkennen, und die Leberstücke sind von schaumiger Flüssigkeit bedeckt. Diese Erscheinungen haben so viel Aehnlichkeit mit den Vorgängen bei der Fäulniss, dass ein früherer Versuch in diesem Stadium ohne weitere Untersuchung verworfen wurde.

48 Stunden nach Beginn des Versuches wird von der Leber auf Bouillon und Agar übergeimpft, ausserdem zur Prüfung auf etwa anwesende Anaëroben eine Stichcultur in das Innere eines Nährbodens angelegt. Alle Nährböden bleiben dauernd steril. 3 )

72 Stunden nach der Leberentnahme wird sodann auch mikroskopisch die absolute Sterilität festgestellt.

1) Die Thiere hatten in diesen Fällen immer einige Stunden nach dem Tode gelegen.

2) Arch. f. exper. Pathol., Bd. XXII, 1886.

3) Die sämmtlichen bakteriologischen Untersuchungen wurden von den Herren Dr. Bruns und Dr. Conradi, Assistenten am Hygienischen Institut, ausgeführt; ich möchte auch an dieser Stelle ihnen für die grosse Freundlichkeit besten Dank sagen. Ganz besonderen Dank schulde ich meinem Freunde, Herrn Dr. Ernst Fuld, der mir mit unermüdlicher Bereitwilligkeit bei den zeitraubenden Thierversuchen half. 
Im Präparate sieht man nur gelbe Klumpen, die wie zerfallenes Lebergewebe aussehen. Bis zum nächsten Tage krystallisiren unter dem Deckglase eine grosse Zahl von typischen Tyrosinbüscheln aus.

Tyrosin wird aber noch deutlicher durch Verarbeitung des ganzen Inhaltes des Röhrchens nachgewiesen. Das Leberstück wird mit wenig destillirtem Wasser gekocht, dann wird filtrirt, das Filtrat eingeengt, der syrupöse Rückstand mit schwachem Alkohol heiss extrahirt.1) Der Alkohol wird dann wieder eingedunstet und der Rückstand über Nacht stehen gelassen. Derselbe zeigt mikroskopisch wiederum typisches Tyrosin und gibt eine sehr intensive Millon'sche Reaction.

Das Resultat dieses Versuches lässt sich in mehrfacher Weise präcis formuliren.

Während Kraus in entsprechend angeordneten Versuchen durch quantitative Bestimmungen den Nachweis führen konnte, dass eine Fettzunahme ${ }^{2}$ ) nicht statthat, ist die Entstehung von Tyrosin in aseptischer Leber durch unseren Versuch erwiesen.

Das ist aber nicht anders möglich, als wenn Eiweisskörper in der «überlebenden» Leber gespalten werden. Damit ist gezeigt, dass die Autolyse nicht nur in Lösungen und unter Zusatz von Antisepticis zu Stande kommt, sondern auch im Lebergewebe, das lediglich aus dem Gesammtorganismus ausgeschaltet ist. So wird es noch wahrscheinlicher, dass wir einen normalen Vorgang, eine physiologische Function der Leberzellen vor uns haben.

Ferner macht der Versuch den Einwand hinfällig, bei unseren antiseptischen Autolyseversuchen könnten wir durch Bakterienwirkungen getäuscht worden sein. Die Möglichkeit eines derartigen Einwandes ist heute dadurch näher gerückt, dass auch proteolytische Enzyme der Bakterien mehrfach gefunden worden sind. Dieser Einwand muss aber fallen gelassen werden, da eben die Autolyse auch bei vollkommen durchgeführter Asepsis zu beobachten ist. Man kann nun auch nicht mehr annehmen, im Lebersaft könne ja Autolyse stattfinden, aber in der histologisch unveränderten Leber seien

1) So haben wir stets am leichtesten das Tyrosin aus der Leber einigermaassen isolirt.

2) Eine Fettbildung wäre immer noch möglich, da sie durch parallel verlaufende Fettzerstörung verdeckt sein könnte. 
vielleicht Substanzen vorhanden, welche diese Wirkung nicht. aufkommen liessen.

Es wäre ferner verfehlt, diesen Eiweisszerfall als eine Absterbeerscheinung der Zellen aufzufassen. Das autolytische Ferment wird durch das Aufhören der Circulation nicht zerstört, und der Unterschied gegenüber dem intravitalen $\mathrm{Zu}$ stand besteht für die Autolyse nur darin, dass jetzt das Material nicht ersetzt wird und die Produkte nicht fortgeschafft werden.

Das sind aber zum Theil sicher dieselben Bedingungen, wie sie bei der aseptischen Nekrose im Sinne der pathologischen Anatomie und bei den embolischen Infarcten gegeben sind. Die proteolytischen Enzyme leiden durch diese pathologischen Ereignisse an sich nicht und können die Rückbildung des ausser Function gesetzten Gewebes besorgen.

In dieser Arbeit haben wir also Anhaltspunkte dafür erhalten, dass die Autolyse der Leber geeignet ist, wichtige Leistungen des intermediären Stoffwechsels zu erklären. Auf die mehrfach gestreifte Beziehung zur Frage der Harnstoffbildung soll noch in einer besonderen Mittheilung eingegangen werden. Wir haben aber ferner gesehen, dass die intracellulären Fermente verdienen, auch zur Erklärung pathologischer Verhältnisse herangezogen zu werden. In einer sich dieser Arbeit unmittelbar anschliessenden Mittheilung wird über Versuche berichtet werden, die sich auf das Verhalten der Autolyse bei der Phosphorvergiftung beziehen. 